\title{
THE MICROWAVE ELECTRO-THERMAL (MET) THRUSTER: A NEW TECHNOLOGY FOR SATELLITE PROPULSION AND ATTITUDE CONTROL
}

\author{
John E. Brandenburg \\ Research Support Instruments \\ Washington Operations \\ 4325-B Forbes Boulevard \\ Lanham, Maryland 20706 \\ Michael M. Micci* \\ Propulsion Engineering Research Center \\ Department of Aerospace Engineering \\ The Pennsylvania State University \\ University Park, PA 16802
}

\begin{abstract}
This paper discusses the current research status of the MET (Microwave ElectroThermal) thruster. In the MET thruster, an electrodeless, vortex stabilized, plasma is produced in a microwave resonator cavity for the purpose of heating gaseous fuel to produce a high temperature rocket exhaust for space propulsion. The higher specific impulse (momentum transfer per unit weight) of these heated gases offers advantages over traditional chemical rockets in terms of reduced fuel mass. In MET devices, dense plasmas have been produced in various possible fuel gases, nitrogen, hydrogen, and ammonia, using 600 to 2200 Watts of microwave power at a frequency of $2.45 \mathrm{GHz}$. Ammonia has been found to give a specific impulse of $550 \mathrm{sec}$. It has been found that the plasma is a $98 \%$ absorber of microwave
\end{abstract}

power leading to negligible reflection of power back to the microwave source and making the cavity operate at low $Q$.

Taking advantage of this effect, it has been found that a very compact MET thruster design could be operated, with the magnetron microwave source and resonator cavity joined in one unit.

\section{Nomenclature}

d length of microwave resonant cavity

e charge of an electron

$E_{O} \quad$ Strength of $E$ field driving a cavity

$f_{c} \quad$ frequency of electron neutral collisions

$f_{p} \quad$ frequency of electron plasma oscillations

$\mathrm{m}_{\mathrm{e}}$ mass of the electron

$\mathrm{n}_{\mathrm{e}} \quad$ electron number density

$\mathrm{N}_{\mathrm{O}}$ neutral particle number density 
Q Quality factor of microwave resonant

$R$ radius of microwave resonant

cavity

$\mathrm{V}_{\mathrm{e}}$ electron thermal speed

$\sigma_{\mathrm{C}} \quad$ electrical conductivity

$\sigma_{\mathrm{o}} \quad$ collision cross section for electrons on neutrals

$\Delta \omega \quad$ full width at half maximum for microwave resonant cavity

$\omega_{0} \quad$ resonant frequency of $\mathrm{TM}_{011}$

mode in microwave resonant cavity

\section{Background}

The MET thruster $(1,2,3)$ is a new electric propulsion technology for space applications and research to develop it is progressing cooperatively at Research Support Instruments, Penn State University and the Center for Space Power at Texas A\&M. Research on the MET thruster has also been pursued at NASA Lewis Research Center( 3 ).

The presently evolved design for the MET thruster (See Figure) consists of a cylindrical microwave resonator cavity tuned to support the $\mathrm{TM}_{011}$ mode, in which a gas vortex is created by tangential injection of gas at high pressure. The use of vortex flows to stabilize free floating microwave discharges was first reported by Kapitsa ${ }^{(4)}$ and was applied to MET first by one of the authors, $M$. Micci. The gas forms a high temperature microwave discharge on the axis of the cavity, at the location of one of the antinodes of the electric field in the $\mathrm{TM}_{011}$ mode, next to an exit nozzle located on the cavity axis. In the MET it is desirable to heat the gas just as it exits the chamber to minimize heat loss to solid structures, thus the cavity is designed so that the plasma is created at the exit nozzle.

The MET demonstrated at RSI operates at $2.45 \mathrm{GHz}$ and $600 \mathrm{~W}$ in the $\mathrm{TM}_{011}$ mode, because this operating point is

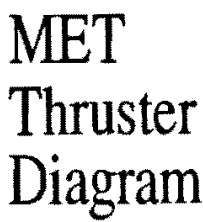

Resonant Cavity

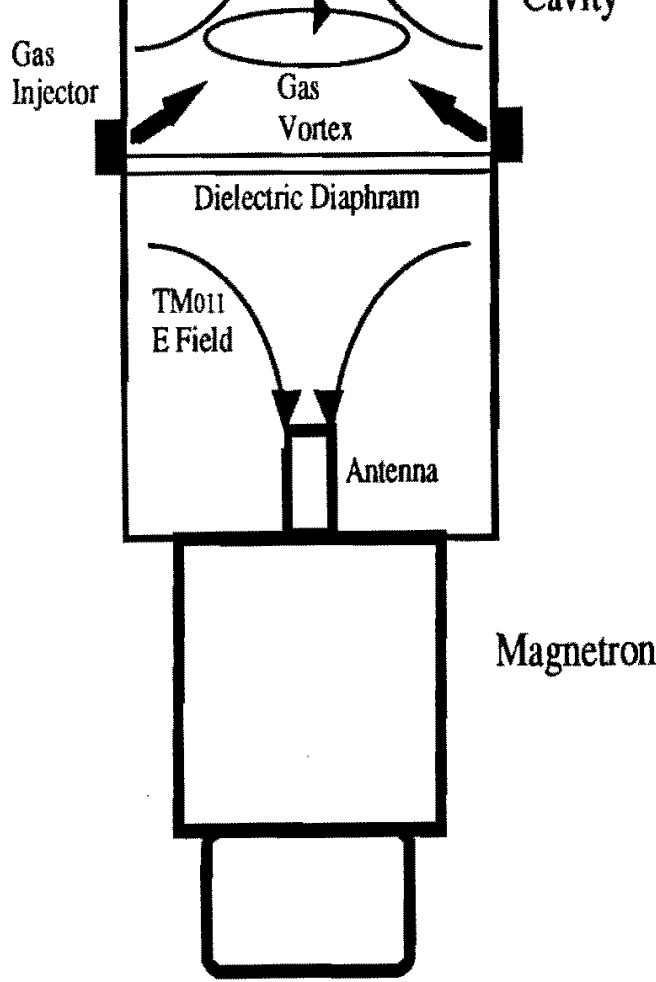

Figure: Diagram of New MET Design (Patent Applied For) 
appropriate for the thruster application on small satellites. However, other frequencies, higher powers, and other modes have also been investigated. Investigators at NASA Lewis Research Center (3) employed $0.915 \mathrm{GHz}$ microwaves to make MET plasmas using both the TM011 and TM012 modes.

When operating in the TM 012 mode, the plasma forms in the center of a cylindrical cavity, away from end or side walls. Using the TM012 mode the workers at NASA Lewis were able to sustain plasmas at up to $11 \mathrm{~kW}$. Operation at higher power was prevented only by the overheating of a confining discharge tube. The $0.915 \mathrm{GHz}$ frequency was chosen because magnetrons operating at $0.915 \mathrm{GHz}$ are remarkably efficient, with $90 \%$ conversion efficiency, making this frequency attractive for high power space applications. Thus, while the research at RSI is presently aimed at perfecting small, low power MET devices, it also appears possible to design large, high power MET thrusters.

The plasma in the MET is very resistive and as a result absorbs approximately 95\% of the incident power, which makes the effective $Q$ of the resonant cavity very low. The resistive character of the MET plasma results from the high gas pressure, 1 atmosphere or above, in which the discharge occurs. In recent research it was discovered that because the cavity has low $Q$, the microwave fields in the cavity can be driven directly by a magnetron generator which has its antenna inserted directly into the cavity. This configuration operates without any apparent loss of efficiency or overheating in the magnetron, and results in an extremely compact thruster design for space applications.

In the remainder of this paper we will discuss the inferred plasma conditions in the MET cavity and how they allow a compact MET design to be realized.

\section{The Phvsics of the MET Cavity}

The MET thruster operates as a heavily damped resonant cavity. The existence of a low order mode in microwave resonant cavities fixes the electric field geometry inside to create a region of strong electric field in the cavity whose location can be controlled. This, in turn, allows the location of the breakdown and the sustained discharge to be controlled. The existence and properties of the plasma in the MET cavity heavily load the cavity, damping the resonant fields and making the conditions of resonance insensitive to the details of the cavity geometry.

Vortex gas flow reinforces the tendency of the plasma to remain in a region of intense electric field in the resonant field and prevents the problem of plasma migration to the microwave antenna, a phenomenon observed in previous experiments without vorticity (3). The presence of vortex flow thus stabilizes the resonant fields by stabilizing the plasma location. Vortex stabilized, low order mode cavities have been run successfully at both $2.45 \mathrm{GHz}$ and $0.915 \mathrm{GHz}$, in both $\mathrm{TM}_{011}$ and $\mathrm{TM}_{012}$ modes. The TM012 mode is similar to the TM011 mode and can be excited in resonators that are only slightly different from those that support TM011. The conditions for resonance in a cylindrical cavity (5) for $2.45 \mathrm{GHz}$ microwaves are:

$$
\begin{aligned}
& \mathrm{TM}_{011}: \mathrm{c} \cdot\left(\mathrm{x}_{01}{ }^{2} / \mathrm{R}^{2}+\pi^{2} / \mathrm{d}^{2}\right)^{1 / 2} \\
& =2.45 \cdot 10^{9} \mathrm{sec}^{-1} \\
& \mathrm{TM}_{012}: \mathrm{c} \cdot\left(\mathrm{x}_{01}{ }^{2} / \mathrm{R}^{2}+4 \pi^{2} / \mathrm{d}^{2}\right)^{1 / 2} \\
& =2.45 \cdot 10^{9} \mathrm{sec}^{-1}
\end{aligned}
$$

where $\mathrm{c}$ is the speed of light, $\mathrm{R}$ is the cylinder radius, $d$ is its length, and $x_{01}=$ 5.520 is the first zero of Bessel function $\mathrm{J}_{\mathrm{O}}(\mathrm{x})$. For the same value of $\mathrm{R}$ in the TM 012 mode, the length $d$ must be twice that used for $\mathrm{TM}_{011}$. In both modes $\mathrm{E}$ 
field lines concentrate at locations along the axis of the cavity creating regions where plasma will form preferentially.

The process of plasma formation in gases by microwaves has been studied extensively, both experimentally and theoretically $(6)$. Because of the high collisional frequency of electrons with neutrals and ions in a discharge at one atmosphere, there is little change in the physics of the discharge between operation at $2.45 \mathrm{GHz}$ or $0.915 \mathrm{GHz}$. This follows from the fact that the electrons in the plasma experience $\mathrm{E}$ field acceleration for much less than one cycle of the external wave field oscillation before they collide with neutrals or ions and scatter. The collision frequency of electrons with neutrals and ions in a 1 atmosphere pressure discharge with electron temperatures of $11,000 \mathrm{~K}(1 \mathrm{eV})$ is approximately found as

$$
f_{c}=\sigma_{o} \cdot N_{o} \cdot V_{e}=10^{11} \sec ^{-1}
$$

where $\sigma_{\mathrm{o}} \sim 1.5 \cdot 10^{-16} \mathrm{~cm}^{2}$ is the cross section for collisions of $1 \mathrm{eV}$ electrons with neutral atoms, $N_{o} \sim 10^{19} \mathrm{~cm}^{-3}$ is the approximate neutral particle density in the discharge at 1 atmosphere, and $\mathrm{V}_{\mathrm{e}} \sim$ $6 \cdot 10^{7} \mathrm{~cm} / \mathrm{s}$ is the thermal velocity of 1 $\mathrm{eV}$ electrons. At $\mathrm{f}_{\mathrm{c}}=10^{11}$, the collision frequency is thus much higher than the microwave frequency even at $2.45 \mathrm{GHz}$. Therefore the plasma electrons experience the wave fields as a series of dc accelerations, rather than an oscillating field.

MET plasmas are inherently absorptive, due to a high rate of electron collisions with neutrals and ions.

The plasmas produced in the MET exhibit visible light extinction lengths of a few millimeters, indicating approximate electron densities of approximately $\mathrm{n}_{\mathrm{e}}=10^{14} \mathrm{e}^{-/} \mathrm{cm}^{3}$. The plasma frequency in this case is approximately

$$
\begin{gathered}
f_{p}=\left(4 \pi e^{2} n_{e} / m_{e}\right)^{1 / 2} / 2 \pi \\
\approx 9000 \cdot n_{e}^{1 / 2}
\end{gathered}
$$

where $\mathrm{e}$ is the electron charge and $\mathrm{m}_{\mathrm{e}}$ is the electron mass, so that $f_{p}$ is approximately $10^{11} \mathrm{~Hz}$. This is approximately the same as the collision frequency, therefore, the electrons in the plasma are therefore dominated by collisional rather than collective effects, and as a consequence resemble high pressure arc discharges $(7)$. The flow of electrons in such a discharge resembles that in an ordinary conductor like carbon, rather than in a collisionless plasma.

Since the electron motion is heavily impeded by collisions, such a discharge is inherently lossy and tends to be an absorber of electromagnetic energy rather than a reflector. At $11,000 \mathrm{~K}$, the conductivity of a plasma in 1 atmosphere gas is found approximately as

$$
\begin{aligned}
& \sigma_{c}=\left(e^{2} n_{e} / m_{e}\right) / f_{c}=f_{p}^{2} /\left(4 \pi f_{c}\right) \\
& \approx 10^{10} \mathrm{~s}^{-1} \text { in esu units }
\end{aligned}
$$

or

$1 \mathrm{mho} / \mathrm{m}$ in MKS units,

which is somewhat lower than the conductivity of carbon at room temperature. Since the plasmas in the MET occupy approximately $10 \%$ of the volume of the cavity, and furthermore occupy that region where most of the electric field energy is stored in the cavity, the resistive plasma exerts a strong damping on the cavity, making it a low $Q$ cavity.

The fact that the value of the plasma conductivity leads to low $Q$ can be seen easily by the expression $(8)$ for $Q$

$\mathrm{Q}=\omega_{\mathrm{o}}$ (Stored energy)/(Power Loss) 


$$
\approx \omega_{\mathrm{o}} \mathrm{E}^{2} /\left(8 \pi \sigma_{\mathrm{c}} \mathrm{E}^{2}\right) \approx \omega_{\mathrm{o}} / 8 \pi \sigma_{\mathrm{c}} \approx 1
$$

where we have used the approximation that the plasma occupies the region where E fields are strongest and thus where most of the E field energy is stored.

The Q of the MET cavity is thus of order unity whereas a normal metal cavity has $\mathrm{Q}$ of the order of 1000 or greater. This has the consequence that the resonance line width of the cavity, the range of frequencies around the fundamental frequency at which the cavity will resonate, is broad being of the order of the frequency.

$$
\Delta \omega=\omega_{0} / Q
$$

This also means that the $E$ field strength in the cavity is approximately the same as the E field driving the cavity which is, the $E$ field near the antenna radiating microwaves into the cavity, since the $\mathrm{E}$ fields in the cavity are related to the driving $E$ fields $E_{0}$ by the relation

$\mathrm{E}=\mathrm{QE}_{\mathrm{O}}$.

Therefore the antenna of the microwaves will see low E fields in the cavity when it radiates and thus microwave power will tend to flow into the cavity and not out of it. The low $Q$ of the MET cavity thus allows the direct application of microwaves from the generator without concern that reflected power will harm the generator. This hypothesis was then verified by experiment, where a magnetron was inserted directly into the cavity and drove the discharge with no measurable change in plasma or magnetron operation.

\section{Summary}

Therefore the operation of the MET thruster cavity is crucially dependent on the presence of the plasma that heats the fuel gas. This plasma transforms the cavity from a high $\mathrm{Q}$ cavity to a low $\mathrm{Q}$ cavity with the attendant effects of low $E$ fields in the cavity, resonance broadening and negligible reflected power. These effects made the direct joining of the cavity to the magnetron possible and successful. The result is a compact design for which a patent has been applied for.

\section{References}

1. S. Whitehair, J. Asmussen, S. Nakanishi, Journal of Propulsion and Power, Vol. 3, p. 136 (1980).

2. D.J. Sullivan, J. Kline, C. Philippe, Micheal M. Micci , AAI-93-2106 AIAA/SAE/ASME/ASEE 31 st Joint Propulsion Conference and Exhibit July 1995.

3. J.L. Power and D.J. Sullivan AAI-93-2106, AIAA/SAE/ ASME/ASEE 29th Joint Propulsion Conference and Exhibit, June 1993, Monterey CA.

4. Kapitsa P.L. Soviet Phys. JETP 30,973-1008 (1970).

5. Om P. Gandhi "Microwave Engineering and Applications" Pergamon Press, New York, p. 248.

6. A.D. McDonald "Microwave Breakdown in Gases " John Wiley and Sons Inc. New York (1966) p. 164.

7. Francis F. Chen "Introduction to Plasma Physics," Plenum Press, New York (1974) p. 12.

8. J.D. Jackson "Classical Electrodynamics 2nd Ed." John Wiley and Sons Inc. New York (1975) p. 357. 